
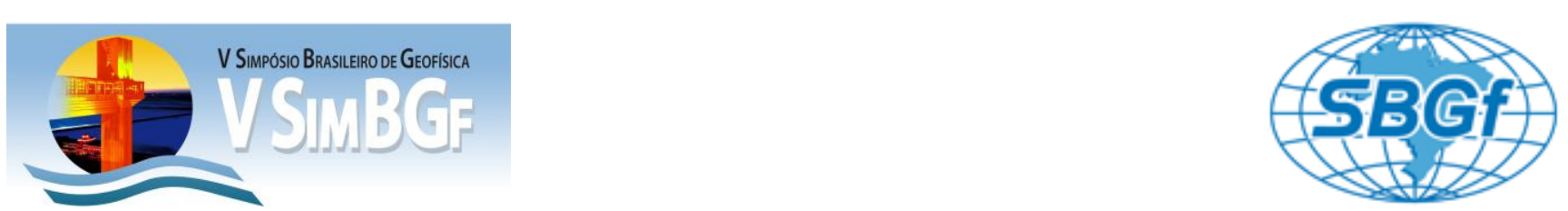

\title{
Processamento e Interpretação de dados aeromagnéticos na porção centro sul da Bacia dos Parecis
}

Faria, H. P. A, Correa, R. T., Vidotti, R. M.; Universidade de Brasília, Instituto de Geociências, Laboratório de Geofísica Aplicada.

Copyright 2012, SBGf - Sociedade Brasileira de Geofísica

Este texto foi preparado para a apresentação no V Simpósio Brasileiro de Geofísica, Salvador, 27 a 29 de novembro de 2012. Seu conteúdo foi revisado pelo Comitê Técnico do V SimBGf mas não necessariamente representa a opinião da SBGf ou de seus associados. É proibida a reprodução total ou parcial deste material para propósitos comerciais sem prévia autorização da SBGf.

\section{Resumo}

A Bacia dos Parecis está entre as maiores bacias intracratônicas brasileiras com uma área de aproximadamente $500 \mathrm{~km}^{2}$. Localiza-se no Centro-Oeste do Brasil, no setor sudoeste do Cráton Amazonas entre a Bacia dos Solimões e a Bacia do Paraná. O desenvolvimento de técnicas de aquisição e processamento de geofísica aérea nas ultimas décadas possibilitou 0 avanço dos estudos de bacias sedimentares e suas estruturas. Técnicas atuais de interpretação se concentram em definir atributos geométricos tanto relacionados a profundidades quanto a estrutura dos corpos magnéticos. Assim, o estudo da Bacia dos Parecis tem como objetivo principal o entendimento de sua geologia, visando também compreender sua estruturação e a profundidade de fontes de magnetização através de levantamentos magnéticos aerotransportados na porção centro sul da bacia. Os resultados permitem caracterizar e diferenciar as anomalias magnéticas da Faixa Paraguai-Araguaia na porção centro sul da área de estudos da Bacia dos Parecis, porção centro norte da área de estudo.

\section{Introdução}

A Bacia dos Parecis está entre as maiores bacias intracratônicas brasileiras com uma área de aproximadamente $500 \mathrm{~km}^{2}$. Localiza-se no Centro-Oeste do Brasil, no setor sudoeste do Cráton Amazonas entre a Bacia dos Solimões e a Bacia do Paraná (Figura 1). A Bacia é descrita por três domínios tectono-sedimentares diferenciados respectivamente pelos altos da Vilhena e da Serra Formosa (Siqueira e Teixeira, 1993). O sul é delimitado pela faixa Paraguai-Araguaia. O extremo oeste é formado por uma depressão tectônica (Fossa Tectônica de Rondônia), a região central é identificada por um baixo gravimétrico da ordem de $-40 \mathrm{mgal}$ (Sub-bacia Juruena) e a região leste é interior tipo sinéclise (Sub-bacia Alto Xingu) (Siqueira, 1989 e Pedreira et al, 2003).

A bacia contém aproximadamente $6 \mathrm{~km}$ de sedimentos do Paleozóico, Mesozóico e Cenozóico e também possui rochas vulcânicas do Cretáceo. Seu embasamento é compreendido de rochas metamórficas de alto e baixo grau, bem como rochas intrusivas. As rochas sedimentares da bacia são ricas em matéria orgânica. É predominantemente preenchida por sedimentos siliclasticos. A presença de algumas camadas de calcário são identificadas entre o Siluriano e o Carbonífero, este último possui frequentes níveis de gipsita e certas lentes de carvão. Alguns kimberlitos são percebidos atravessando a sequencia sedimentar, mesmo que a manifestação magmática dominante do cretáceo tenha ocorrido em suas margens (Siqueira, 1989)

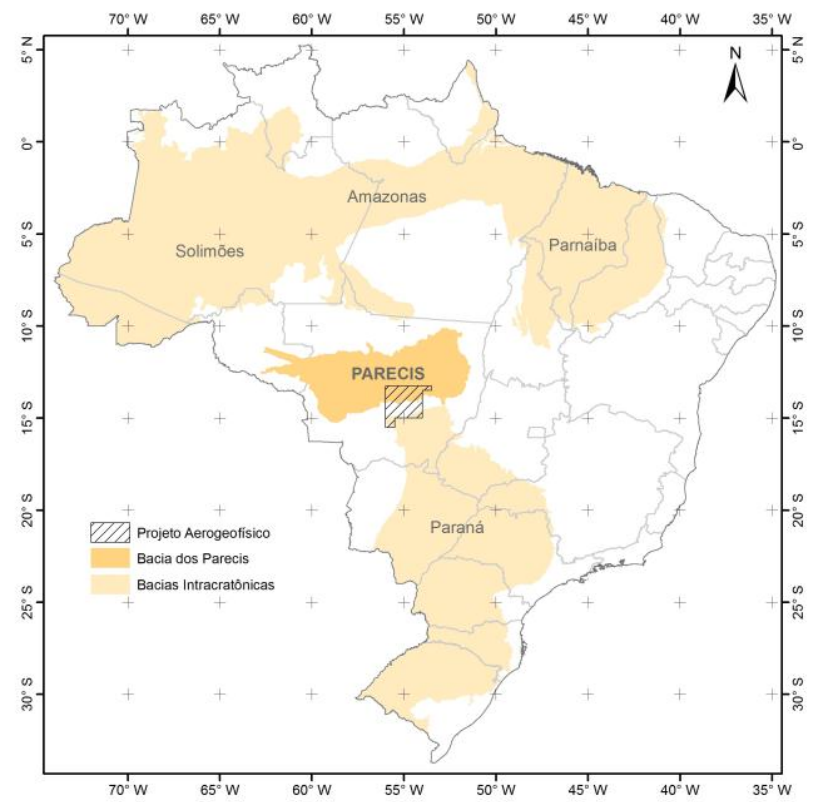

Figura 1 - Mapa de localização da área de trabalho. Os limites das bacias intracratônicas foram extraídos de Bizzi et al, 2003.

Segundo Gunn (1997), o desenvolvimento de novas tecnologias na aquisição de dados de geofísica aérea possibilitou uma maior relevância destes nos estudos de bacias sedimentares e suas estruturas, revelando a existência de gradientes magnéticos surgindo de estruturas sedimentares, visto que anteriormente apenas a identificação do embasamento das bacias sedimentares era possível. Técnicas atuais de interpretação se concentram em definir atributos geométricos tanto relacionados a profundidades quanto a estruturas dos corpos magnéticos.

Assim, o estudo da Bacia dos Parecis tem como objetivo principal o entendimento de sua geologia, visando também compreender sua estruturação e a profundidade 
de suas fontes de magnetização através de levantamentos magnéticos aerotransportados na porção centro sul da bacia.

A interpretação dos mapas geofísicos consolidou uma base para uma análise estrutural de escala regional. Tal base é confirmada através do método da deconvolução de Euler e da amplitude do sinal analítico.

\section{Materiais e Métodos}

Os dados utilizados nesse trabalho foram cedidos para o Instituto de Geociências da Universidade de Brasília (IGUnB) pelo Serviço Geológico do Brasil (CPRM) para utilização acadêmica. O levantamento magnético, Projeto Mato Grosso - Área 1 (código 1072) cobre a borda centro sul da Bacia dos Parecis (Figura 1), com área de, aproximadamente, $46.257 \mathrm{Km}^{2}$, onde a extensão dos perfis alcançou $98.400 \mathrm{~km}$ lineares. As linhas de voo estão na direção Norte-Sul espaçadas em 500 metros e as de controle com direção L-W e espaçamento de 5 quilômetros.

Os dados foram cedidos para o IG/UnB pré-processados no formato GDB, possibilitando a confecção do mapa do Campo Magnético Anômalo (CMA) através do algoritmo de mínima curvatura, com malha regular de $125 \mathrm{~m}$. Todavia o mapa se apresentava com muito ruído na direção das linhas de voo em todos os produtos derivados do CMA. Desta forma, para melhorar a relação sinal/ruído, foi necessária a utilização de algumas técnicas de filtragem. Utilizou-se um micronivelamento, com o objetivo de suavizar as linhas de voo sem que haja mudanças nas características do dado. O algoritmo do micronivelamento utilizado é composto por dois filtros consecutivos: Butterworth e cosseno direcional. O filtro Butterworth utiliza a formula:

$$
L(k)=1 / 1+{ }_{\left(\frac{k}{k 0}\right)^{n}},
$$

onde k0 é o numero de onda central; e $n$ é a ordem do filtro.

O cosseno direcional utiliza a formula:

$$
L(\theta)=\left|\cos ^{n}(\alpha-\theta+\pi / 2)\right|,
$$

onde a é o azimute a partir do norte e $n$ é o grau da função.

Butterworth é um filtro simples e eficaz, pois pode controlar o grau de roll-off enquanto o comprimento de onda central é mantido fixo (Geosoft, 2005). Seus parâmetros ajustáveis são Cutoff Wavelenght $(1 / \mathrm{k} 0)$, Filter Order e High or Low Pass. Foram feitos vários testes e os melhores resultados foram obtidos com os parâmetros $1 / \mathrm{k} 0=200, \mathrm{n}=8$ e utilizando Low Pass.

O filtro do cosseno direcional é muito eficiente para remover ruídos direcionais do grid. Ele foi utilizado para atenuar o ruído na direção das linhas de voo eliminando o excesso de informações contidas nesta direção (Cordell et al., 1992).

Os dados foram processados no software Oasis Montaj da Geosoft $\circledast$, versão 7.0.2, integrados e interpretados no ArcGis da $E S R I \cap$, disponíveis IG/UnB.

\section{Resultados}

Com os dados processados e micronivelados foi possível gerar o mapa do campo magnético anômalo (CMA) sem a presença de ruídos na direção das linhas de voo (Figura 2). Posterior à obtenção do CMA foram gerados outros produtos, sendo eles suas derivadas horizontais de primeira ordem na direção $x$ e $y$, e sua derivada vertical de primeira ordem, denominadas $\partial x, \partial y$ e $\partial z$ respectivamente.

As derivadas são normalmente aplicadas ao campo magnético para realçar as anomalias mais rasas e podem ser calculadas tanto no domínio do espaço quanto na frequência. Entretanto, esse operador amplifica o ruído de alta frequência.

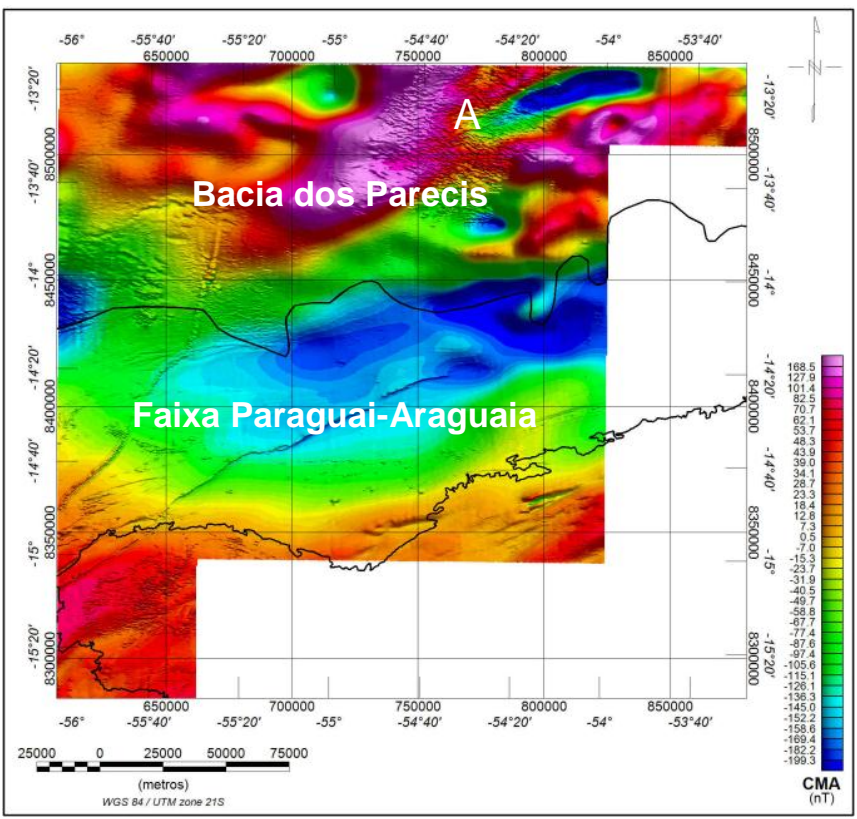

Figura 2- Campo Magnético Anômalo, a linha preta na porção norte da figura representa o limite sul da Bacia dos Parecis; a linha preta ao sul indica o limite norte da bacia do Paraná, entre as duas bacia localiza-se a Faixa Paraguai.

Os produtos $\partial x, \partial y$ e $\partial z$ foram diferenciados com 0 intuito do calculo da Amplitude do Sinal Analítico (ASA). O sinal analítico é conhecido por e atenuar os longos e ampliar os curtos comprimentos de onda do sinal geofísico nas direções dos três vetores ortogonais do espaço, contrastando as bordas e os contatos de feições geológicas e falhas (Nabighian, 1972, 1974; Roest et al, 1992). 


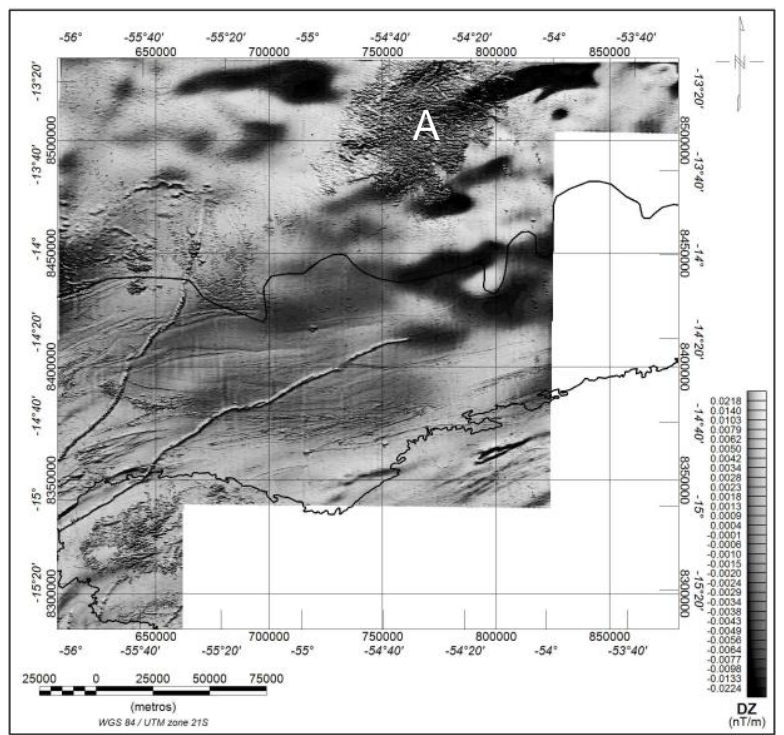

Figura 3 - Derivada vertical de primeira ordem.

A Amplitude de sinal analítico é calculado através da seguinte formula:

$$
|A|=\sqrt{\left(\frac{\partial T}{\partial x}\right)^{2}+\left(\frac{\partial T}{\partial y}\right)^{2}+\left(\frac{\partial T}{\partial z}\right)^{2}}
$$

Onde A é a amplitude do sinal analítico, T é o campo geomagnético medido em $\{\mathrm{x}, \mathrm{y}\}, \partial x, \partial y$ e $\partial z$ se referem as derivada nas direções $x, y$ e $z$, respectivamente (Nabighian, 1972, 1974; Roest et al, 1992).

A ASA é um produto importante para encontrar bordas de corpos magnéticos particularmente onde a remanência e/ou latitude magnética baixa dificulta a interpretação. Pela forma resultante da ASA, as soluções são obtidas como as localizações $(x, y, z)$ da fonte. A distância entre os pontos de inflexão do sinal analítico anômalo é diretamente proporcional à profundidade do topo da fonte magnética, o que pode ser observado na figura 4. O sinal analítico pode ser utilizado para delinear os contrastes magnéticos e dar estimativas de profundidades aproximadas (e.g. Reynolds, 1997).

Juntamente com a ASA, o método de deconvolução de Euler foi utilizado para auxiliar na interpretação da área em questão. Tal método é uma inversão que calcula a profundidade do topo dos corpos que possuem características magnéticas apresentadas no levantamento (Thompson, 1982). Também opera no dado diretamente e proporciona uma solução matemática sem recorrer a nenhuma restrição baseada na geologia, o que é um ponto positivo, pois não está ligado à nenhuma ideia geológica pré-concebida. Este fato pode ser usado criticamente para avaliar as interpretações geológicas.

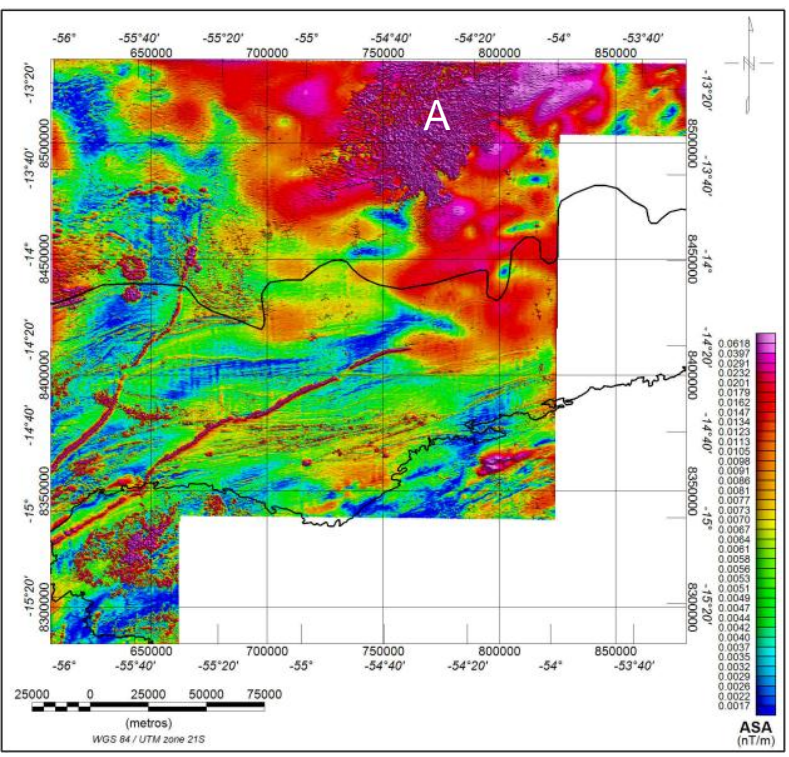

Figura 4 - Amplitude do Sinal Analítico.

A equação do método de deconvolução de Euler (Thompson, 1982; Reid et al. 1990) é dada por:

$$
\left(x-x_{\mathrm{o}}\right) \frac{\partial T}{\partial x}+\left(y-y_{\mathrm{o}}\right) \frac{\partial T}{\partial y}-\left(z-z_{\mathrm{o}}\right) \frac{\partial T}{\partial z}=N(B-T),
$$

Onde $x_{\circ}, y_{\circ}$ e $z_{o}$ são as coordenadas da fonte magnética cuja intensidade do campo magnético $T$ e seu valor regional $\mathrm{B}$ são medidos no ponto $(\mathrm{x}, \mathrm{y}, \mathrm{z})$; $\mathrm{N}$ é o grau de homogeneidade e se refere ao índice estrutural. Nas figuras 5 e 6 foi utilizado o índice estrutural com valor 1 , que representa feições lineares, bidimensionais.

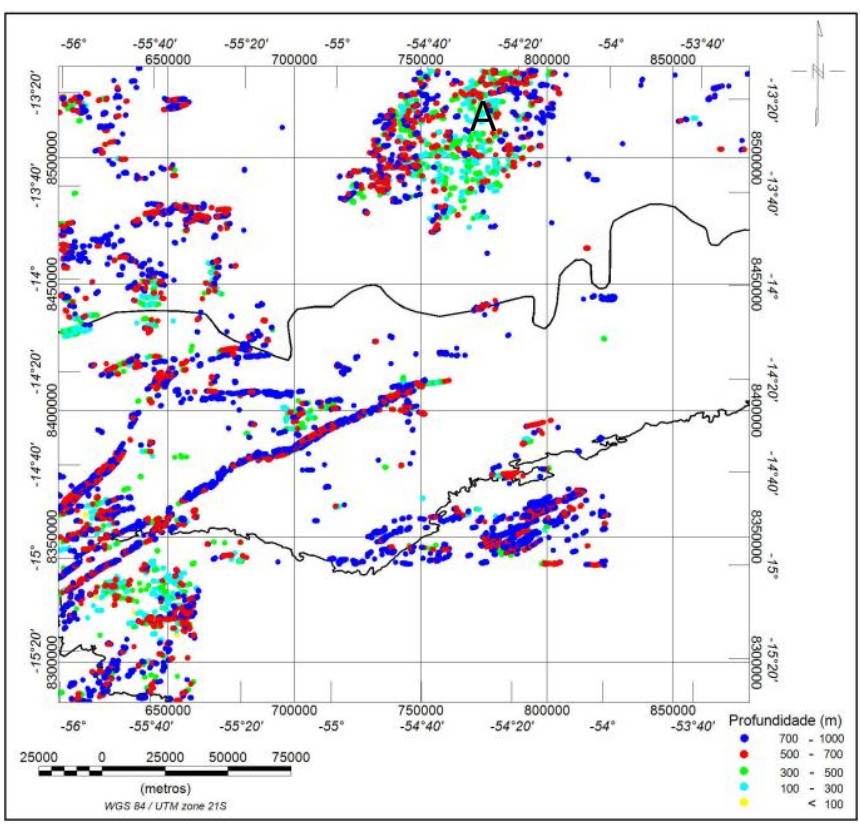

Figura 5- Resultados da deconvolução de Euler com variação de profundidade entre 0 e 1000 metros. 
Como o resultado consiste em determinar profundidades de fontes magnéticas, leva-se em consideração um erro estatístico relacionado à máxima tolerância de profundidade. No caso dos dados utilizados neste trabalho adotou-se um valor de $5 \%$. Soluções que ultrapassaram esse limite foram rejeitadas.

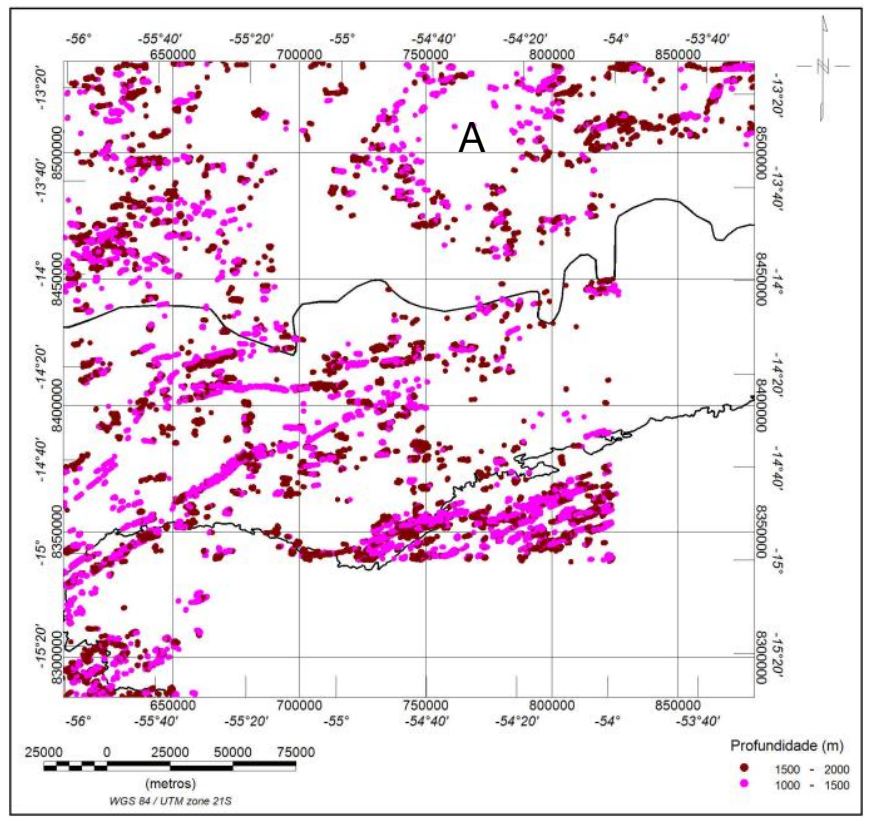

Figura 5- Resultados da deconvolução de Euler com variação de profundidade entre 1000 e 2000 metros.

\section{Discussão e Conclusões}

A partir do mapa do campo magnético anômalo (figura 2) e do sinal analítico (figura 4) é possível identificar na parte central dos mapas um baixo magnético com comprimento de onda de cerca de $50 \mathrm{~km}$, que está relacionado a Faixa Paraguai-Araguaia (Hasui et al, 1977). Nas figuras 2, 3 e 4 percebe-se a existência de anomalias lineares e continuas a partir da margem oeste dos mapas com direção nordeste. As profundidades estimadas para estas anomalias variam de 500 a 2000 metros, sendo que de 700 a 1500 metros as profundidades apresentam continuidade. De 500 a 700 e acima de 1500 metros não há continuidade. Algumas dessas anomalias se estendem em direção a Bacia dos Parecis segundo o limite adotado nesse trabalho. Dentro da Bacia a anomalia tem direção NNE com profundidades mais rasas próximo à borda (de 100 a 700 metros) e mais profundas ao norte (de 700 a 1500 metros).

A porção norte do mapa é caracterizada por altos magnéticos cujas anomalias tem cerca de 50 a $100 \mathrm{~km}$ de extensão, o que pode representar altos do embasamento da bacia. A porção centro norte dos mapas (A nas figuras 2, 3 e 4) possui uma região restrita, de aproximadamente $500 \mathrm{~km}^{2}$, caracterizada por altos magnéticos com alta frequência podendo indicar intrusões ígneas.
Essas anomalias são evidenciadas na deconvolução de Euler (A, figura 5) a corpos de mais rasos, com profundidades variando de 100 a 500 metros. Localmente observa-se profundidades de 500 a $1000 \mathrm{~m}$ e nas bordas (A, Figura 6) profundidades maiores que $1000 \mathrm{~m}$.

Analisando o ASA (figura 4) nota-se anomalias isoladas características de kimberlitos. Essas anomalias apresentam profundidades variáveis a partir de $100 \mathrm{~m}$ (figura 6).

Em todos os produtos obtidos e analisados nota-se uma variação nas características magnéticas entre a Bacia dos Parecis e a Faixa Paraguai-Araguaia, porém, não há anomalias características ou resultados na deconvolução de Euler que permitam a correlação destas com o limite geológico adotado neste trabalho.

O progresso deste trabalho envolverá a integração e interpretação de outros levantamentos aerogeofísicos (magnéticos, gravimétricos e gamaespectrométricos) disponíveis para a execução deste estudo.

\section{Agradecimentos}

A divisão de Geofísica do Serviço Geológico do Brasil (CPRM) pela cessão dos dados geofísicos do Projeto Mato Grosso, Área 1 (código 1072), À ESRI pela disponibilização do Pacote de ferramentas que compõem a Família ArcGis 10 por intermédio do contrato $N^{\circ} 2011$ MLK 8733 e a IMAGEM pelo apoio e viabilidade da concretização do termo de uso entre o IG-UnB e a ESRI e pelo suporte aos softwares. Ao Laboratório de Geofísica Aplicada do IG/UnB pela infraestrutura para o desenvolvimento deste trabalho.

\section{Referências}

Bizzi, L. A.; Schobbenhaus, C.; Vidotti, R. M.; Gonçalves, J. H. (Orgs). Geologia, Tectônica e Recursos Minerais do Brasil - Texto, Mapas e SIG. , 2003. Brasília, CPRM. $674 p$.

Cordell L., Phillipes J.D \& Godson R.H. 1992. US geological Survey potential-Field Geophysical software =, version 2.0 USGS,Open-File Report 92.18p.

Geosoft. 2005. Montaj Geophysics v6.1.Tutorial and User Guide.

Gunn P.J., Maidment D. \& Milligan P.R. 1997. Interpreting aeromagnectic data in areas of limited outcrop. AGSO, Journal of Australian Geology e Geophysics.,17:175-186.

Hasui, Y., Abreu, F.A.M., Silva, J.M.R. 1977. Estratigrafia da faixa de dobramentos Paraguai-Araguaia no centronorte do Brasil. B. Inst. Geoc. USP. São Paulo, 8:107118.

Nabighian, M. N., 1972., The analytic signal of two dimensional magnetic bodies with polygonal cross-section 
- Its properties and user for automated anomaly interpretation: Geophysics, 37: 507-517.

Nabighian, 1974, Additional comments on the analytic signal of two-dimensional magnetic bodies with polygonal cross-section: Geophysics, 39: 85-92.

Pedreira A. J. \& Lopes R. C. \& Vasconcelos A. M. Bahia. R. B.C. 2003. Bacias Sedimentares Paleozóicas e MesoCenozóicas. In: Bizzi, L. A.; Schobbenhaus, C.; Vidotti, R. M.; Gonçalves, J. H. (Orgs). Geologia, Tectônica e Recursos Minerais do Brasil - Texto, Mapas e SIG. , 2003. Brasília, CPRM. p 55-85.

Reid, A. B., J. M. Allsop, H. Granser, A.J. Millet, and I. W. Somerton, 1990, magnetic interpretation in three dimensions using Euler deconvolution: Geophysics, 55: 80-91.

Reynolds J.M. 1997. An introduction to applied and environmental geophysics. John Wiley \& Sons, $796 \mathrm{p}$.

Roest , W. R., j. Verhoef, and M. Pinlkington, 1992, Magnetic interpretation using the $3 D$ analytic signal: Geophysics, 57: 116-125

Siqueira L.P. 1989. Bacia dos Parecis. Boletim de Geociências da Petrobrás, 3:3-16.

Siqueira L.P. \& Teixeira L.B. 1993. Bacia dos Parecis: nova fronteira exploratória da Petrobrás.SBGeof., Congr. Intern. Soc. Bras. Geof.

Thompson, D.T, 1982, EULDPH - A new techinique for making computer-assisted depth estimate from magnetic data: Geophysics, 47: 31-37. 